\title{
Performance Analysis on Channel Estimation with Antenna Diversity of OFDM Reception in Multi-path Fast Fading Channel
}

\author{
Hyun Seo $\mathrm{Oh}^{1} \cdot$ Do Wook Kang ${ }^{1}$ \\ Published online: 13 August 2018 \\ (C) The Author(s) 2018
}

\begin{abstract}
Vehicle to everything (V2X) communication supports vehicle to anything communication for vehicle safety and cooperative Intelligent Transport System in vehicular environments. IEEE 802.11p modem has been developed and applied for V2X communication system. $\mathrm{V} 2 \mathrm{X}$ radio channel has multipath fast fading due to moving vehicles and surrounded road structure. We proposed a new DFCE-AD which combines DFCE and antenna diversity for OFDM reception and analyzed the performance improvement in multipath fading channel. Through computer simulation, SNR gain of DFCE-AD over DFCE for QPSK modulation is approximately $6 \mathrm{~dB}$ at PER $=10 \%$. In other word, PER of DFCE-AD is improved over that of DFCE by about $20 \%$ at SNR $=10 \mathrm{~dB}$. This result will be applied for the short sized packet and low order OFDM modulation in vehicular multipath fading channel.
\end{abstract}

Keywords V2X · WAVE - Multi-path fading channel - Decision feedback channel estimation (DFCE) - Decision feedback channel estimation with antenna diversity $(\mathrm{DFCE}-\mathrm{AD}) \cdot$ Packer error rate $(\mathrm{PER})$

\section{Introduction}

As Information and Communication Technology (ICT) is advanced, vehicles are connected to surrounding vehicles and road infrastructure by using wireless connectivity. The wireless connectivity enables vehicles to recognize neighboring vehicle's moving status or road's situation and provide vehicle safety and Cooperative Intelligent Transport System (C-ITS). Thus vehicle's wireless connectivity will contribute on reduction of traffic

This work was supported by Electronics and Telecommunications Research Institute (ETRI) grant funded by the Korean government [17ZS1500, High Speed V-Link Communication Technology Development for Real Time Control of Autonomous Driving Vehicle].

Hyun Seo Oh

hsoh5@etri.re.kr

Do Wook Kang

kdw4653@etri.re.kr

1 Autonomous Driving System Research Group, Electronics and Telecommunications Research Institute, Daejeon 305-700, South Korea 
accident, congestion and $\mathrm{CO}_{2}$ emission [1, 2]. $\mathrm{V} 2 \mathrm{X}$ connectivity provides vehicle to anything communication in urban or high way environments. V2X radio channel has multipath fast fading due to moving vehicles and surrounded road structure [3-6]. In dense urban intersection, a public bus and a small car may be mixed in moving and vehicle to vehicle wireless channel may have Non Line of Sight propagation with multipath propagation. Also in highway driving, vehicle to vehicle channel may have deep fading due to the reflected interference of the neighboring heavy truck or fast fading due to the high relative speed in moving vehicles with the opposite direction. There was much research on V2X radio channel model. Georgia technology has published $5.9 \mathrm{GHz}$ radio channel modeling through experiment and theoretical analysis. And V2X radio channel has Non Line of Sight (NLOS) multipath fading characteristics in vehicular environment [4-6]. NLOS multipath fading channel model can be modeled as Jakes channel model [7-9].

Wireless Access in Vehicular Environments (WAVE) communication adopted Orthogonal Frequency Division Multiplexing (OFDM) modulation with $10 \mathrm{MHz}$ channel spacing and increased RF power for the extension of radio coverage. It supports Binary Phase Shift Keying (BPSK), Quadrature Phase Shift Keying (QPSK), 16 Quadrature Amplitude Modulation (16 QAM) or 64 Quadrature Amplitude Modulation (64 QAM) and convolutional coding scheme $[10,11]$. The received OFDM signal through NLOS multipath fading channel will have fast change in the amplitude and phase term. To improve the performance of OFDM reception, many channel estimation techniques have been studied. The pilot symbol may be inserted into the data symbol to estimate fast change of channel phase $[12,13]$. And the position of pilot signals may be changed [14]. Proposed schemes may improve the channel estimation. However, they need to change the signal form of transmitted OFDM signal. The channel estimation schemes are proposed; channel estimation by using inter-symbols averaging in time domain, channel estimation by using inter-symbols correlation in time domain, channel estimation by using data symbol feedback [12-16].

The decision feedback channel estimation scheme has excellent estimation performance in fast fading channel among many channel estimation techniques. In this paper, we propose a new channel estimation method using Decision Feedback Channel Estimation with Antenna Diversity (DFCE-AD). For the given OFDM signal format, we analyzed the PER performance in the multipath fading channel if conventional Decision Feedback Channel Estimation (DFCE) scheme is applied. Also, we analyze PER performance of proposed DFCE-AD scheme.

In the Sect. 2, we introduce V2X OFDM system model. It consists of OFDM transmitter, multipath fading channel model and receiver. In Sect. 3, the proposed channel estimation scheme is described. In Sect. 4, computer simulation and performance results are discussed. Finally the conclusions are summarized in Sect. 5.

\section{V2X OFDM System Model}

The V2X OFDM system consists of OFDM transmitter, multipath fading channel model and receiver as shown in Fig. 1. Packet Data Generation sends randomly generated data to OFDM Modulator and Packet Error Count measures the number of packet error of the received packet data from the receiver. OFDM Modulator generates OFDM modulated signal and insert training sequence for initial packet detection and synchronization [10]. The modulated signal $\mathrm{X}$ will be distorted in amplitude and phase through multipath fading channel. The multipath fading channel can be modeled as Tapped Delay Line (TDL) filter. 


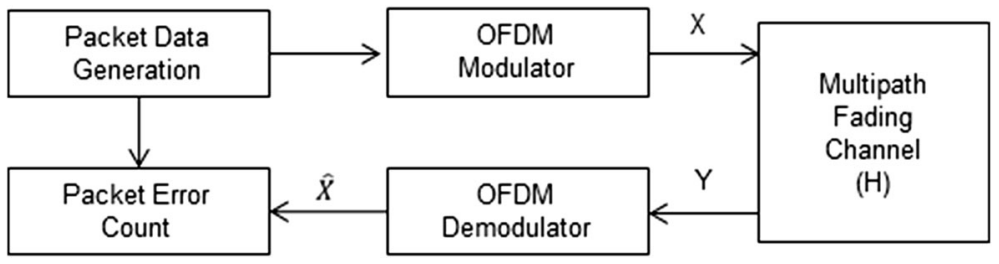

Fig. 1 OFDM system model

The received signal $\mathrm{Y}$ through multipath fading channel will be input to OFDM demodulator to recover data $\hat{X}$.

OFDM demodulator in Fig. 2 detects the start time of received packet by correlating the received training sequence with the internal reference sequence. After packet detection and Fast Fourier Transform (FFT) processing, Least Square (LS) Estimator generates Channel Estimate $\hat{H}$ by using the reference signal of the received signal. The OFDM signal of IEEE $802.11 \mathrm{p}$ has a reference signal in the head part of packet and does not have continuous reference signal. Therefore, the recovered data symbol $\hat{X}$ is mapped and scrambled to be used as the continuous reference signal for channel estimation. LS estimator is applied because it is simple to implement and other optimal estimator such as MMSE may be applied.

The recovered signal will be simply expressed by the Eq. (1).

$$
\hat{\mathrm{X}}=\hat{H} \cdot Y
$$

\section{Proposed Decision Feedback Channel Estimation with Antenna Diversity (DFCE-AD)}

The OFDM signal of IEEE 802.11p has a reference signal in the head part of packet and only supports initial channel estimation. The DFCE scheme is an effective method to provide continuous channel estimation. We propose a new DFCE-AD which combines DFCE and antenna diversity because antenna diversity is known as an effective way for vehicular fading channel. In this scheme, initial channel estimation and the continuous channel estimation is the same as the conventional scheme except for the digital combining using antenna diversity. The digital combining may have equal gain combining, path selection combining and maximal ratio combing and it is well known that selection

$\mathrm{RX}$

Antenna

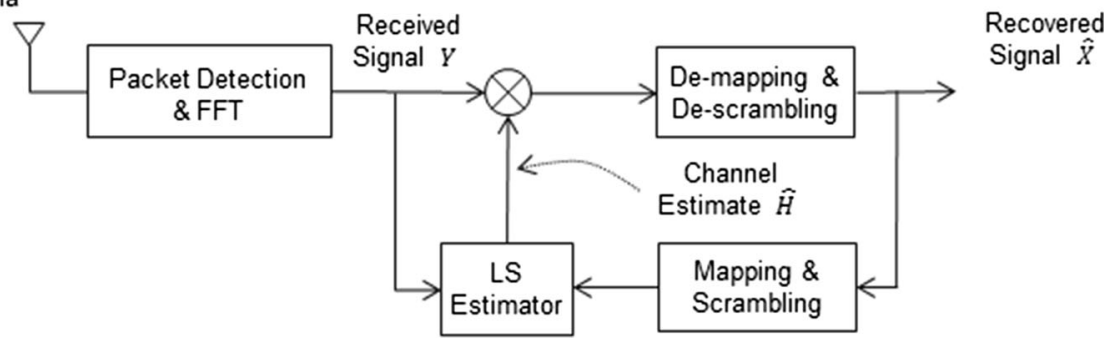

Fig. 2 Conventional decision feedback channel estimation 
combining or maximal combining has a good performance in multipath fading channel. And the channel estimation and digital combining are processed by every OFDM symbol.

The recovered signal will be simply expressed by the Eq. (2) and the below Fig. 3 shows system configuration of DFCE-AD scheme.

$$
\hat{\mathrm{X}}=\hat{H}_{1} \cdot Y_{1}+\hat{H}_{2} \cdot Y_{2}
$$

\section{Performance Analysis}

The performance analysis has been conducted by computer simulation. The simulation modeling consists of packet generation, OFDM signal and multipath fading channel, the receiver and packet reception in Table 1. In the performance analysis, packet size and mobility are considered as the key simulation parameters because DFCE-AD algorithm has data feedback channel estimation type and impacted by packet size and multipath mobility.

Packet generation will be able to generate short packet or long packet because vehicle safety applications has short packet which is an order of 300 bytes and IP applications has long packet which is an order of 1000 bytes. OFDM signal format will have BPSK, QPSK, 16 QAM and 64 QAM scheme and multipath fading channel will have Doppler spectrum in $140 \mathrm{~km} / \mathrm{h}$ or $400 \mathrm{~km} / \mathrm{h}$ moving condition. Practically $140 \mathrm{~km} / \mathrm{h}$ mobility considers highway driving and $400 \mathrm{~km} / \mathrm{h}$ assumes that two vehicles are moving in the opposite direction and the relative speed will be doubled.

At the receiver, we have applied DFCE or DFCE-AD scheme. And we calculated PER for the given signal-to-noise ratio (SNR).

Computer simulation has taken 10,000 iterations to calculate PER under the given conditions by using MATLAB.

Figure 4 shows performance result of conventional DFCE for modulation level BPSK, QPSK, QPSK and 64 QAM in the condition of packet size 300 bytes and $140 \mathrm{~km} / \mathrm{h}$ vehicular mobility. The required SNR at PER $=10 \%$ is analyzed because the minimum PER of IEEE 802.11p standard is specified to $10 \%$. To meet PER $=10 \%, \mathrm{SNR}=15 \mathrm{~dB}$ for BPSK, SNR $=16 \mathrm{~dB}$ for QPSK and $16 \mathrm{QAM}, \mathrm{SNR}=25 \mathrm{~dB}$ for $64 \mathrm{QAM}$ are

$\mathrm{RX}$

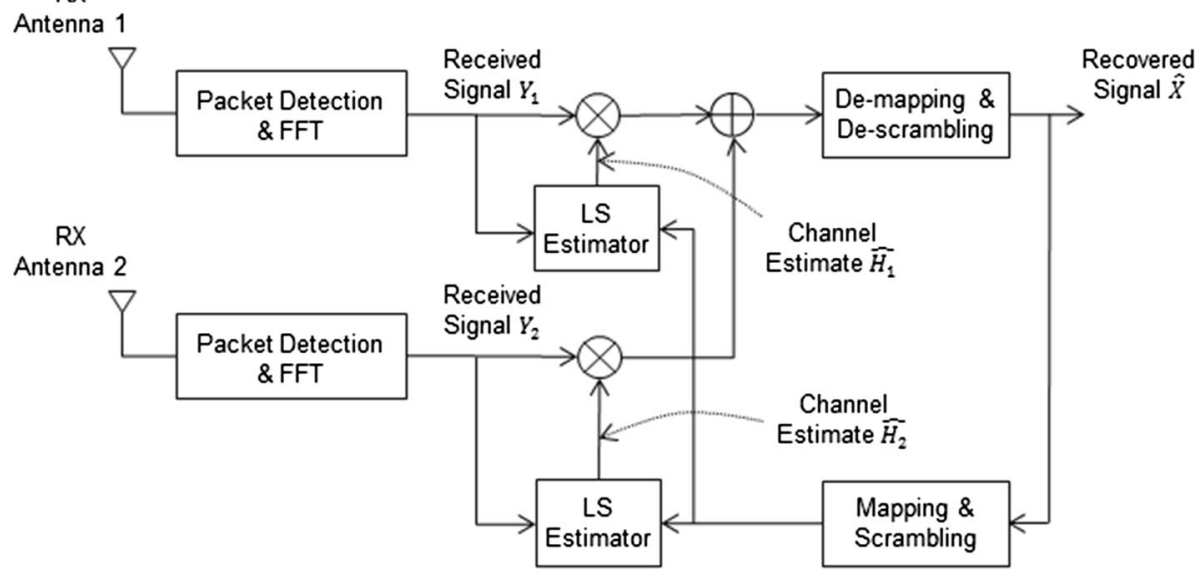

Fig. 3 Proposed decision feedback channel estimation DFCE-AD 
Table 1 Computer simulation modeling

\begin{tabular}{ll}
\hline Item & Simulation parameter \\
\hline Packet size & 300 byte, 1000 byte \\
Modulation level & BPSK, QPSK, 16 QAM, 64 QAM \\
Fading channel & TDL with $140 \mathrm{~km} / \mathrm{h}$ or $400 \mathrm{~km} / \mathrm{h}$ mobility \\
Channel estimation method & DFCE, proposed DFCE-AD (maximal ratio combining) \\
SNR & $1-25 \mathrm{~dB}$ \\
\hline
\end{tabular}

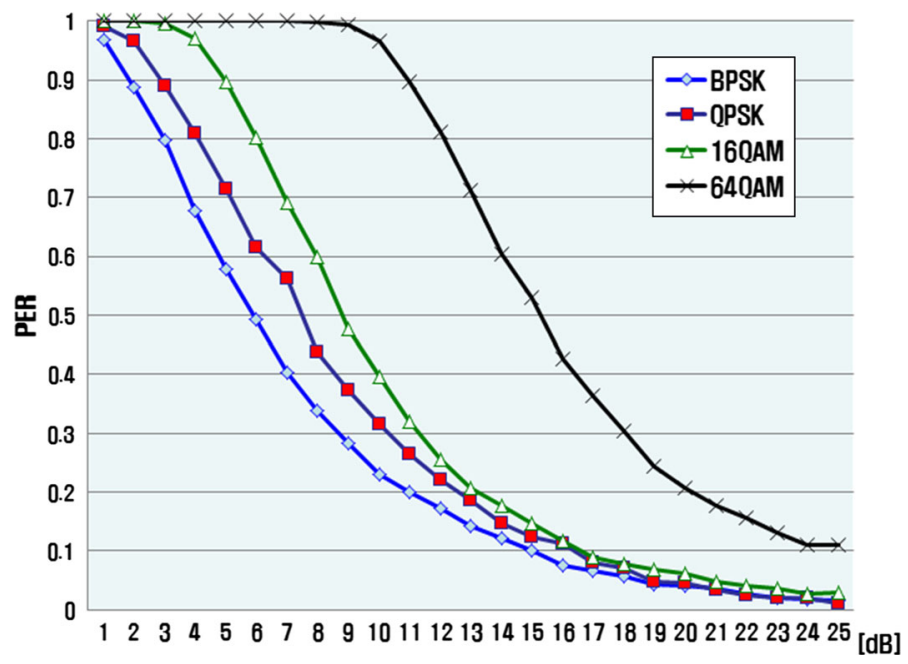

Fig. 4 PER of DFCE with packet size $=300$ bytes in $140 \mathrm{~km} / \mathrm{h}$ mobility

approximately required. And Fig. 5 shows performance result of proposed DFCE-AD for modulation level BPSK, QPSK, QPSK and 64 QAM in the same condition of Fig. 4. To meet PER $=10 \%$, SNR $=8 \mathrm{~dB}$ for BPSK, SNR $=10 \mathrm{~dB}$ for QPSK, SNR $=12 \mathrm{~dB}$ for $16 \mathrm{QAM}, \mathrm{SNR}=19 \mathrm{~dB}$ for $64 \mathrm{QAM}$ are required. From Figs. 4 and 5, SNR gain of DFCE-AD over DFCE will be approximately $7 \mathrm{~dB}$ in BPSK, $6 \mathrm{~dB}$ in QPSK, $4 \mathrm{~dB}$ in 16 QAM, $6 \mathrm{~dB}$ in 64 QAM. Figure 6 shows performance improvement of DFCE-AD over DFCE for QPSK modulation in the condition of packet size 300 bytes and $140 \mathrm{~km} / \mathrm{h}$ vehicular mobility. From the Fig. 6, SNR gain of DFCE-AD over DFCE is approximately $6 \mathrm{~dB}$ at PER $=10 \%$. And PER of DFCE-AD is improved over that of DFCE by about $20 \%$ at $\mathrm{SNR}=10 \mathrm{~dB}$.

We analyzed the impact on the performance of DFCE-AD which is related to the packet size and the mobility. As for the sample cases; $140 \mathrm{~km} / \mathrm{h}$ or $400 \mathrm{~km} / \mathrm{h}, 300$ bytes or 1000 bytes, the performance is analyzed.

In Fig. 7, SNR of $400 \mathrm{~km} / \mathrm{h}$ mobility at PER $=10 \%$ will be degraded over that of $140 \mathrm{k} / \mathrm{h}$ mobility by $6 \mathrm{~dB}$. In other words, PER performance of $400 \mathrm{~km} / \mathrm{h}$ mobility at $\mathrm{SNR}=16 \mathrm{~dB}$ is degraded over that of $140 \mathrm{~km} / \mathrm{h}$ mobility by about $10 \%$.

In Fig. 8, SNR of 1000 bytes at PER $=10 \%$ will be degraded over that of 300 bytes by about $4.5 \mathrm{~dB}$. In other words, PER performance of 1000 bytes at $\mathrm{SNR}=16 \mathrm{~dB}$ is degraded 


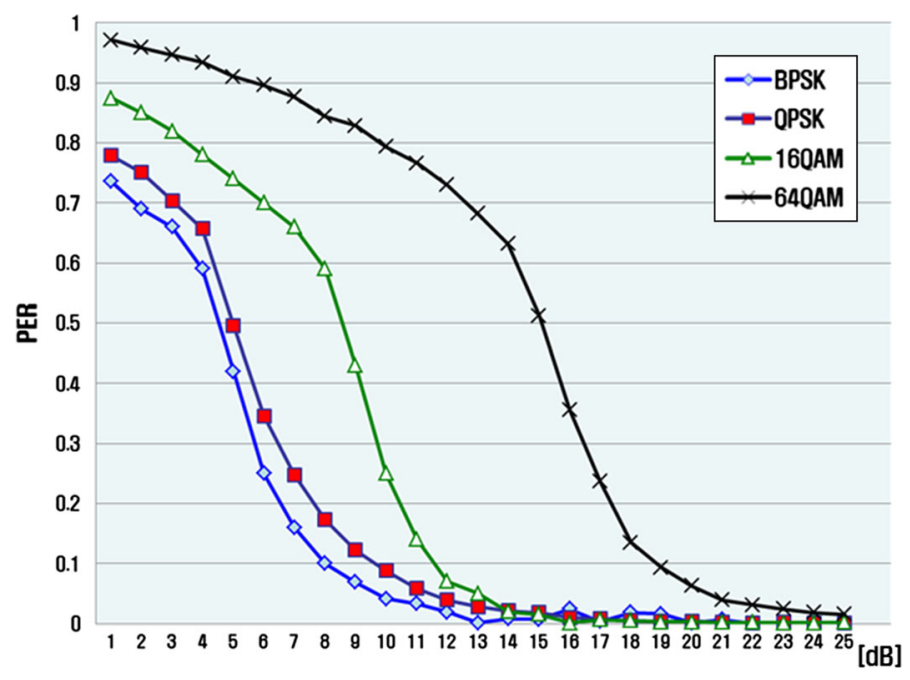

Fig. 5 PER of proposed DFCE-AD with packet size $=300$ bytes in $140 \mathrm{~km} / \mathrm{h}$ mobility

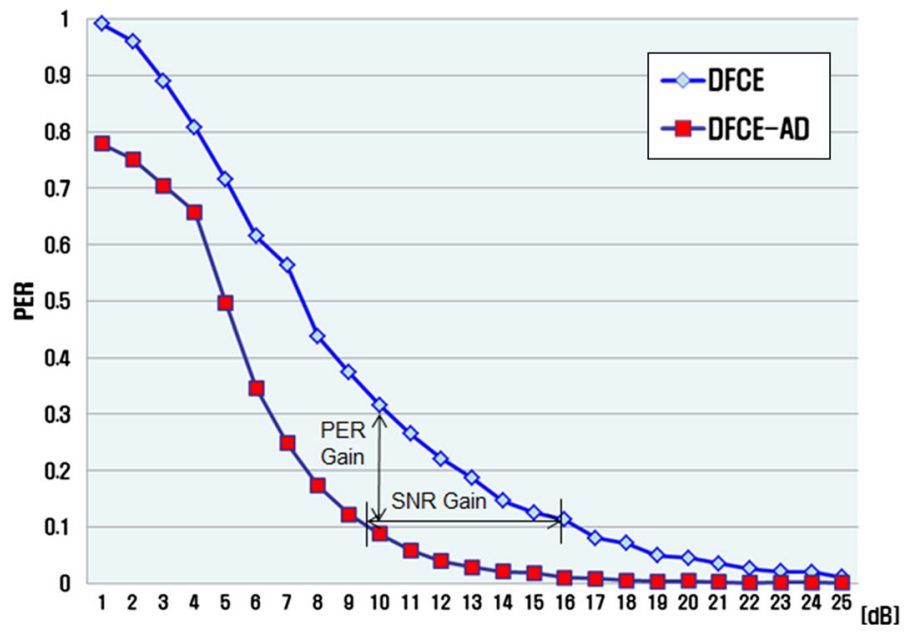

Fig. 6 Performance improvement of DFCE-AD over DFCE for QPSK with packet size $=300$ bytes in $140 \mathrm{~km} / \mathrm{h}$ mobility

over that of 300 bytes by about $15 \%$. This tendency shows that the performance of DFCE$\mathrm{AD}$ is directly related to the mobility and packet size.

From the computer simulation, the proposed DFCE-AD has have SNR improvement on the conventional DFCE scheme for packet transmission of the low order modulation. And the PER of the short sized packet or low order modulation signal is not impacted by the vehicle mobility. However, PER of the long sized packet or high order modulation signal is impacted.

DFCE-AD algorithm had been implemented into ASIC logic and performance test had been conducted at $100 \mathrm{~km} / \mathrm{h}$ driving in highway environment. When OFDM transceiver was mounted on test vehicle and 8000 packets with 1000 bytes were transmitted, PER at 


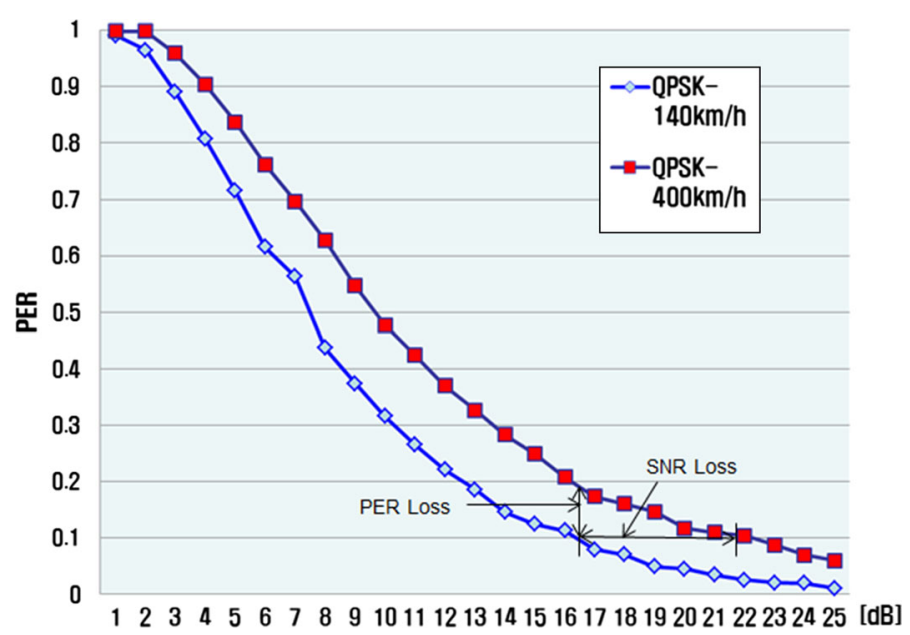

Fig. 7 Performance of DFCE-AD in $140 \mathrm{~km} / \mathrm{h}$ or $400 \mathrm{~km} / \mathrm{h}$ mobility

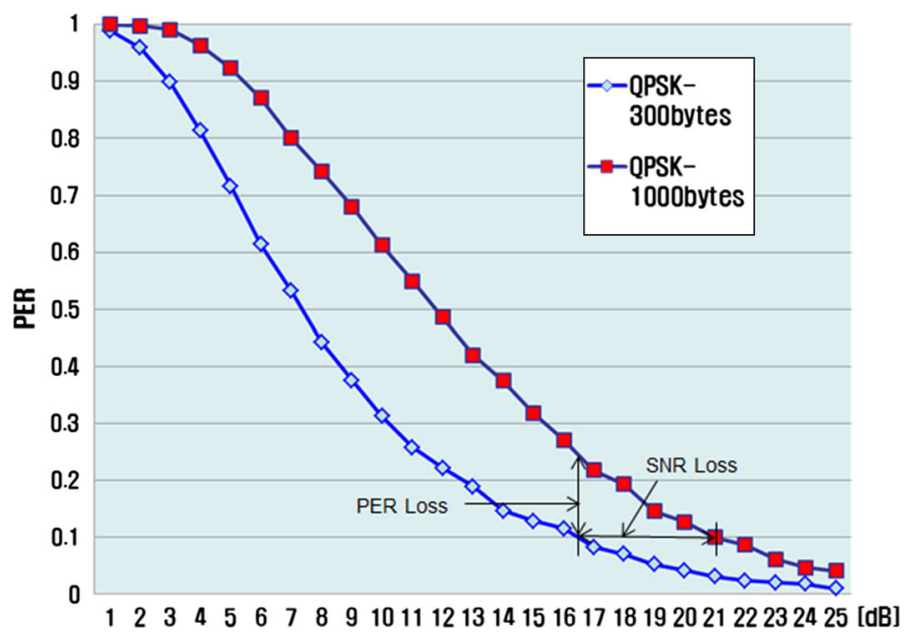

Fig. 8 Performance of DFCE-AD with packet size $=300$ bytes or 1000 bytes

the receiver was counted for DFCE and DFCE-AD schemes respectively. Field test result showed that PER of DFCE-AD is better than that of DFCE in highway environment by about $20 \%$.

\section{Conclusion}

V2X communication supports vehicle to anything communication for vehicle safety and cooperative Intelligent Transport System in vehicular environments. IEEE 802.11p transceiver has been used for V2X applications.

We proposed DFCE-AD in IEEE 802.11p ODDM reception and analyzed PER performance through computer simulation and filed test. From our analysis, there is the performance improvement of DFCE-AD over DFCE by about $6 \mathrm{~dB}$ at PER $=10 \%$ for the 
low order modulation such as BPSK, QPSK and 16 QAM. This means that there is PER improvement by $20 \%$ at the given SNR. Also it was verified that the proposed DFCE-AD has PER improvement by about $20 \%$ over DFCE for QPSK modulation through the field test.

Open Access This article is distributed under the terms of the Creative Commons Attribution 4.0 International License (http://creativecommons.org/licenses/by/4.0/), which permits unrestricted use, distribution, and reproduction in any medium, provided you give appropriate credit to the original author(s) and the source, provide a link to the Creative Commons license, and indicate if changes were made.

\section{References}

1. Vehicle-to-Vehicle Communications: Readiness of V2V Technology for Application, NHTSA, August, 2014.

2. TNO, "Overview of standards for first deployment of C-ITS", Nov. 2014.

3. Sen, I., \& Matolak, D. W. (2008). Vehicle-vehicle channel models for the 5-GHz band. IEEE Transactions on Intelligent Transportation Systems, 9(2), 235-245.

4. Acosta-Marum, G., \& Ingram, M. A. (2007). Six time-and frequency-selective empirical channel models for vehicular wireless LANs. IEEE Vehicular Technology Magazine, 2(4), 4-11.

5. Acosta-Marum, G. (2007). Measurement, modeling, and OFDM synchronization for the wideband mobile-to-mobile channel. Ph.D. dissertation, Georgia Inst. Technol., Atlanta, GA.

6. Wang, C. X., Cheng, X., \& Laurenson, D. I. (2009). Vehicle to vehicle channel modeling and measurements: Recent advances and future challenges. IEEE Communication Magazine, 47(11), 96-103.

7. Jakes, W. C. (1994). Microwave mobile communications. New York: IEEE Press.

8. Sampei, S. (1997). Applications of digital wireless technologies to global wireless communications. Upper Saddle River, NJ: Prentice-Hall.

9. Hiroshi, H. (2002). Simulation and software defined radio for mobile communications. Norwood: Artech House.

10. IEEE 802.11p. IEEE Standard for Information Technology Telecommunication and Information Exchange between Systems Local and Metropolitan Area Networks Specific Requirements. Part II: Wireless LAN MAC and PHY Specifications Amendment 6: Wireless Access in Vehicular Environments, July 22, 2014.

11. ETSI ES 202 663, Intelligent Transport Systems (ITS); European profile standard for the physical and medium access control layer of Intelligent Transport Systems operating in the $5 \mathrm{GHz}$ frequency band, V.1.1.0, 2010.

12. Kim, S. I., Oh, H. S., \& Choi, H. K. (2008). Mid-amble aided OFDM performance analysis in high mobility vehicular channel. In Proceedings of intelligent vehicles symposium, 2008 (pp. 751-754).

13. Cho, W., Kim, S. I., Choi, H. K., Oh, H. S., \& Kwak, D. Y. (2009). Performance evaluation of V2V/V2I communications: The effect of mid-amble insertion. In Proceedings of 1st international conference on wireless communication, vehicular technology, information theory aerospace electronic systems technology (pp. 793-797).

14. Fernandez, J. A., Borries, K., Cheng, L., Vijaya Kumar, B. V. K., Stancil, D. D., \& Bai, F. (2012). Performance of the $802.11 \mathrm{p}$ Physical layer in vehicle-to-vehicle environments. IEEE Transactions on Vehicular Technology, 61(1), 3-14.

15. Zhao, Z., Cheng, X., Wen, M., Jiao, B., \& Wang, C.-X. (2013). Channel estimation schemes for IEEE 802.11p standard. IEEE Intelligent Transportation Systems Magazine, 5(4), 38-49.

16. Alexander, P., Harley, D., \& Grant, A. (2010). Cooperative intelligent transport system: 5.9 GHz field trials. In Proceeding of IEEE (pp. 1-23).

Publisher's Note Springer Nature remains neutral with regard to jurisdictional claims in published maps and institutional affiliations. 


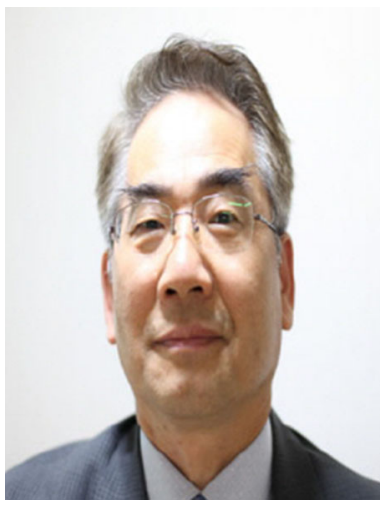

Hyun Seo Oh is a principal researcher leading national project on V2X communication for vehicle safety and C-ITS applications. Dr. Hyun Seo Oh received the B.S. degree in electronic engineering from Soongsil University in 1982, and M.S. degree in electronic engineering from Yonsei University in 1985. And he received Ph.D. in electronic engineering from Yonsei University in 1998 and has been visiting researcher in Ohio State University (OSU), USA. He has joined the research staff of ETRI in 1982. Then, he worked on the system engineering of digital switching system and secure communication system which has block ciphering and stream ciphering. Also he has developed cellular systems such as IS-95, PCS and IMT-2000 system. He have joined ITS (Intelligent Transport Systems) project to develop $5.8 \mathrm{GHz}$ DSRC packet communication system for ETC and smart antenna project to develop adaptive antenna techniques for WCDMA cellular system and TDD-CDMA. Recently he is leading vehicle to vehicle (V2V) and vehicle to infrastructure (V2I) communication technology for automated driving system. He has been committee member of Korea ITS society and invited editor in IEEE vehicular communication society. He has published more than 150 journal papers and patents in vehicular communication and its applications.

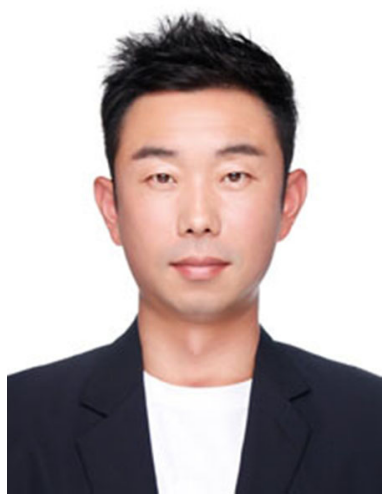

Do Wook Kang received the B.S. and M.S. degrees in Electrical and Electronic Engineering from Dongshin University, Korea, in 2002 and 2004 , respectively. Since 2011, He was a principal researcher in the R\&D Center, Fumate Co., Ltd. He is currently the senior engineer of the autonomous driving system research Group, Electronics and Telecommunications Research Institute (ETRI). He has participated in the development of pico-cell-based high-speed wireless backhaul system. Also he has developed a ground penetrating radar system for safety inspection of transportation infrastructure. His research interests include future mobile networks, vehicular communications, channel modeling, and intelligent transportation systems. 